\title{
EXUDATION AND ABSORPTION MECHANISMS OF THE NORMAL CONJUNCTIVAL CAPILLARIES*
}

BY

\author{
K. H. GO
}

\section{Formosa}

IT is still not clear how the cornea which has no vessels in its tissue is nourished, but the problem of the nutrition of tissue is closely related to the vascular structure, so that if we wish to solve the problem of corneal nutrition we must examine the construction and functions in normal and abnormal situations of the finest conjunctival capillaries at the limbus terminating in the end-capillaries.

Following up this point, before entering upon the study of the cornea, I examined the end-capillaries of the limbus in viro with a slit lamp to discover their construction and function. A special enquiry was made into the exudation and absorption mechanisms, and the results of these investigations are presented below.

\section{Material and Technique}

The construction, blood stream, tonus, and exudation and absorption mechanisms of the end-capillaries of the limbus were examined by inserting a drop of dionine $0.5-3.0$ per cent., pilocarpine $0.5-1.0$ per cent., histamine $0.5-1.0$ per cent., adrenaline 5000 , and atropine $0.1-1.0$ per cent.

The temperature of the solutions was that of normal blood heat. The patients were young men ( 15 to 25 years old) with no disease of the eyes. The slit lamp was not very brightly illuminated and the field of the microscope was irradiated with the reflex light coming from the surface of the iris.

\section{Results}

The original anatomical construction of the end-capillaries is the same $a^{\text {ls }}$ that of the capillaries in the skin papillae. They end in a bow shape and have one arterial and one venous shank. Sometimes they have two or three venous shanks; but the arterial capillaries never terminate at the end of $\mathrm{f}^{\mathrm{f}}$ the arterial shank but have a returning venous shank. It is easy to distinguish both types of shank by the flow of blood.

The blood streams in the capillaries are not permanent and sometimes the capillaries contain no blood. The various blood streams may be seen in the same field of vision of the microscope. Eight types of capillary were distinguished:

* Received for publication June 30, 1952. 
A physiological oedema of the epithelium (Vogt, 1931) may be seen in the peripheral portion of the normal cornea. This phenomenon is caused by the infusion of lymph coming from the end-capillaries.

\section{(1) Instilling one drop Dionine (3 per cent.)}

The capillaries dilated promptly and all now contained a blood stream. The blood streams changed to the rapid continuous non-granular type.

After 2 minutes the fine grey-white spots were beginning to ooze from the arterial shanks and then from the venous shanks and transitional portions. The shank seemed just like a stamen with its pollen. The oozing gradually spread to the nearby tissue, and was thick especially at the branched portion. After about 25 minutes on an average, the exudation reached the maximum, the region of the capillaries was diffusely muddy and was lined from the clear cornea for a little way from the top of the shanks. After about 30 minutes the arterial shanks contracted and the blood streams grew slower and more granular, sometimes static. After about 60 minutes the region of the capillaries grew clearer, the arterial shanks were dilating, the stasis disappeared, and the blood streams were becoming quicker and more granular. After about 120 to 150 minutes, the absorption mechanism had ceased to operate and everything became normal.

\section{(2) Instilling one drop Histamine (1 per cent.)}

The capillaries dilated promptly. The blood streams changed to the rapid continuous non-granular type. After 20 to 30 minutes the Epithelbetaung of the region of the capillaries grew clearer, but the fine grey-white spots did not appear. After about 30 minutes, the arterial shanks were contracting a little and the blood streams became slower and more granular, sometimes static. Then the arterial shanks became dilated, and the blood streams grew quicker, and after about 100-120 minutes everything became normal.

\section{(3) Instilling one drop Pilocarpine (1 per cent.)}

The capillaries dilated promptly, and the blood streams changed to the rapid continuous non-granular type, but the fine grey-white spots did not appear, and the Epithelbetauung was not clearer than that seen with histamine. After 30-50 minutes the effect of the pilocarpine wore off and everything became normal.

(4) Influence on the Action of Dionine of Adrenaline (5000X), and of Eserine (1 per cent.), Atropine (1 per cent.), or Cocaine (1 per cent.)

(a) Dionine plus Adrenaline.-About 60 per cent. of the capillaries dilated slowly, but at different times. Some of the arterial shanks were contracted, blood free, and static. In the dilated capillaries the blood streams changed to the rapid continuous non-granular type. After 2-3 minutes the fine grey-white spots appeared from the dilated capillaries, but not from those which were unchanged or constricted.

(b) Dionine plus Eserine (1 per cent.), Atropine (1 per cent.), or Cocaine (1 per cent.) The capillaries dilated whether the dionine was instilled before or after the eserine, atropine, or cocaine. The exudation appeared, and the effect of the dionine was not disturbed. 


\section{Discussion}

(1) The results show that by instilling one drop dionine the mechanism of exudation and absorption from the capillaries can easily be set in motion within 2 minutes. The exudation-period can be distinguished from the absorption-period as follows:

(i) During exudation the capillary is dilated, and the blood stream is rapid.

(ii) During absorption the arterial capillary is slightly contracted and the blood stream is slow at first; then both gradually return to normal.

Histamine causes only slight exudation from the capillary and does not cause the grey-white spots of oozing.

Pilocarpine causes hardly any exudation from the capillary.

Adrenaline can hinder the action of dionine, but atropine, eserine, and cocaine do not.

(2) For a histological examination of the fine grey-white spots we can refer to Nakamura (1937), who examined a section of the ocular and pericorneal conjunctival tissue after inserting one drop dionine. The epithelium of the conjunctival tissue was swollen, and the conjunctival capillaries were dilated and filled with leucocytes just like the lymph-capillaries. The leucocytes not only filled the capillaries but penetrated the nearby tissue. About 90 per cent. were eosinophil leucocytes.

The fine grey-white spots are therefore eosinophil leucocytes, and the Epithelbetauung are the Epithelödem from the end-capillaries (Vogt, 1931).

(3) The capillary must be dilated in order to cause exudation, and slight contraction of the arterial capillary always causes absorption. This is clearly shown if adrenaline is used to hinder the action of dionine. Krogh (1929) reports the same results in the examination of animals, in that when the blood stream is fast the exudation appears and when it is slow absorption occurs. Krogh describes how the arrested blood stream causes an increase of the permeability of capillaries, and Ricker and Regendanz (1921), who examined the conjunctival vessels of the rabbit, describe how when the blood stream is arrested the exudation does not occur and the postrubrostatischer Zustand is the same as the leucodiapedetischer Zustand. They say that the leucodiapedesis can be seen only in the larger vessels with an axial stream and a wall stream; it is never seen in the capillaries, and it occurs only when the blood stream is slow.

(4) The function of the vessels is governed by the vasomotor nerves. Adrenaline, atropine, pilocarpine, and cocaine do not cause exudation from the capillaries, and adrenaline hinders it even when the exudation mechanism has been operating. If one drop of adrenaline be instilled during absorption, the arterial shanks, already slightly contracted, contract spasmodically and the absorption mechanism has more time for its recovery process than when 
dionine only is used. It appears that this slight contraction of the arterial capillaries during absorption is caused by the sympathetic nerve being slightly stimulated by the exudate. Adrenaline can excite the endings of the sympathetic nerves, and pilocarpine and atropine have an effect upon the para-sympathetic nerves. These do not cause exudation. Therefore both exudation and absorption are caused by the alteration of the nature of the capillary wall. The vasomotor nerves have no primary or active effect upon the capillaries in these processes.

\section{Summary}

When one drop of dionine is instilled into the conjunctival capillary the following results are observed:

(1) The exudation mechanism is set in motion when the capillary is dilated, and the blood stream is fast.

(2) The absorption mechanism acts through the venous shank of the capillary when the arterial shank is slightly contracted and the blood stream is slow or static.

(3) Both mechanisms are caused by an alteration in the capillary wall which is effected by the direct action of specific substances.

(4) The leucodiapedesis caused by dionine appears from the capillary as a grey-white spot during exudation, and returns through the venous shank of the capillary during absorption.

\section{REFERENCES}

Cohnheim, J. (1867). Virchows Arch. path. Anat., 40, 1.

Krogh, A. (1929). "The Anatomy and Physiology of Capillaries ", 2nd ed. Yale University Press, New Haven.

Landis, E. M. (1927). Amer. J. Physiol., 82, 217.

Müller, O. (1937-39). "Die feinsten Blutgefasse des Menschen”, vols. 1 and 2. Enke, Stuttgart.

Nakamura, Y. (1937). Acta. Soc. opthal. jap., 41, 104.

Ricker, G., and Regendanz, P. (1921). Virchows Arch. path. Anat., 231, 1.

VOGT, A. (1931). "Lehrbuch und Atlas der Spaltlampen-mikroskopie des lebenden Auges", 2nd ed. Springer, Berlin. 